\title{
Partial caries removal may have advantages but limited evidence on restoration survival
}

\author{
Abstracted from \\ Ricketts D, Lamont T, Innes NP, Kidd E, Clarkson JE. \\ Operative caries management in adults and children. \\ Cochrane Database Syst Rev 2013; 3: CD003808. doi: 10.1002/14651858.CD003808.pub3 \\ Address for correspondence: Luisa Fernandez Mauleffinch, Review Group Co-ordinator, \\ Cochrane Oral Health Group, MANDEC, School of Dentistry, University of Manchester, \\ Higher Cambridge Street, Manchester, M15 6FH, UK. E-mail: luisa.fernandez@manchester.ac.uk
}

Question: In unrestored primary and permanent teeth with dentinal caries, what are the effects of stepwise, partial or no dentinal caries removal compared with complete caries removal?

Data sources Cochrane Oral Health Group's Trials Register, CENTRAL, Medline via OVID, EMBASE via OVID; no restrictions on language or date of publication.

Study selection Parallel group and split mouth randomised and quasi-randomised controlled trials comparing stepwise, partial and no dentinal caries removal with complete caries removal in unrestored primary and permanent teeth were included in this review.

Data extraction and synthesis Title and abstract screening was by two reviewers, with disagreements resolved by a third. Full texts of eligible studies were assessed by the team until consensus, and data extraction was by three reviewers independently and in triplicate. Two reviewers assessed risk of bias. Trial authors were contacted where possible. Results Eight trials (all assessed as high risk of bias) with 934 participants and 1372 teeth were included in this updated review (Previously complete or ultraconservative removal of decayed tissue in unfilled teeth, Ricketts, 2006) with four new trials being included. There were a number of different comparisons in the trials (stepwise or partial or no dentinal caries removal compared to complete caries removal) with one study including more than one of these comparisons. Four studies investigated primary teeth, three permanent teeth and one included both.

For stepwise caries removal, (four studies), there was a $56 \%$ reduction in incidence of pulp exposure (RR $0.44,95 \% \mathrm{Cl} 0.33$ to $0.60, \mathrm{P}$ $<0.00001$ ) compared to complete caries removal. The mean pulp exposure incidence was $34.7 \%$ in the complete caries removal group and $15.4 \%$ in the stepwise groups. There was no difference in signs and symptoms of pulp disease ( $R R 0.78,95 \% \mathrm{Cl} 0.39$ to $1.58, \mathrm{P}=0.50$ ).

In the two partial caries removal studies, the incidence of pulp exposure reduction was $77 \%$ for the partial caries removal group (RR 0.23 , $95 \% \mathrm{Cl} 0.08$ to $0.69, \mathrm{P}=0.009$ ) with a mean pulp exposure incidence of $21.9 \%$ in the complete caries removal groups and $5 \%$ in the partial

This paper is based on a Cochrane Review published in the Cochrane Library 2013, issue 3 (see www.thecochranelibrary.com for information). Cochrane Reviews are regularly updated as new evidence emerges and in response to feedback, and the Cochrane Library should be consulted for the most recent version of the review. caries removal groups. There was insufficient evidence to determine whether or not there was a difference in signs and symptoms of pulp disease (RR $0.27,95 \% \mathrm{Cl} 0.05$ to $1.60, \mathrm{P}=0.15$ ), or restoration failure (one study showing no difference and another study showing no failures in either group).

There were two very different studies which looked at no dentinal caries removal compared to complete caries removal. There was some evidence of no difference between these techniques for the outcome of signs and symptoms of pulp disease and reduced risk of restoration failure, favouring no dentinal caries removal, from one study. There were no instances of pulp disease or restoration failure in either group from the second study. Meta-analysis of these two studies was not carried out because of the substantial clinical differences between the studies. Conclusions For management of dentinal caries, both stepwise and partial excavation showed clinical advantage over complete caries removal by reducing the incidence of pulp exposure in symptomless, vital, carious primary as well as permanent teeth. The review found no difference in signs or symptoms of pulpal disease between stepwise excavation and complete caries removal.

There was insufficient evidence to determine whether there was a difference in signs and symptoms of pulp disease or a difference in the risk of restoration failure with partial caries removal.

For the two no dentinal caries removal studies, the one investigating permanent teeth found no difference in restoration failure and the one investigating primary teeth found a statistically significant difference in restoration failure favouring the intervention.

Due to the short term follow-up, low reporting of patient centred outcomes and high risk of bias, further high quality, long-term clinical trials are still required to assess the most effective intervention.

\section{Commentary}

Dental caries in children and adults persists as a major public health issue in most communities worldwide. For many clinicians, the technical management of caries has not altered greatly since the time of GV Black, despite the introduction of adhesive materials and concepts such as minimal intervention dentistry (MID).

Traditionally the surgical management of caries has involved the excision of altered tooth structure; however the concept of the complete cleansing of the tooth has been challenged over the past few decades. Three main techniques have been advocated: 1) stepwise excavation; 2) partial caries removal and 3) no dentinal caries removal.

The justification for reducing the amount of 'carious' tooth structure excised (especially dentine) has centred on reducing 
the physical damage to the tooth, which may have an impact on the structural integrity of the tooth, and decreasing the chance of pulpal exposure.

An initial concept from the work of Massler ${ }^{1}$ and Fusayama ${ }^{2}$ was the removal of only infected/decomposed dentine, however difficulty and uncertainty exists around clinically determining what is affected/demineralised or infected/decomposed dentine. The affected dentine has the ability to remineralise under a well placed and sealed restoration, and so can be left in situ. 3,4

The avoidance of exposure of pulpal tissue by removal of carious dentine potentially avoids complicated treatment. ${ }^{5}$ And long-term prognosis for pulpal health may relate more to the status of the pulp at time of initial treatment rather than the technique used for dentine removal and whether an exposure occurs and what material is placed onto it. ${ }^{6,7}$

The objectives of this review were to assess the effects of stepwise excavation, partial caries removal and no dentinal caries removal when compared to complete caries removal. It is an update of a 2006 review ${ }^{8}$ including four additional trials.

Eight trials were included, with 934 participants and 1372 teeth. Four trials investigated primary teeth, three permanent teeth and one both primary and permanent. Four trials related to stepwise technique compared to complete caries removal; in three partial compared to complete caries removal; and in two no dentinal removal was compared to complete removal. One trial had three arms comparing complete caries removal to stepwise and partial caries removal.

The primary outcomes were exposure of the dental pulp during caries removal, signs or symptoms of pulpal disease, progression of caries and restoration failure.

With respect to pulpal exposure, marked reduction in exposure rates occurred with stepwise and partial caries removal when compared to complete caries removal (56\% and 77\% respectively). Interestingly, there was no difference in the pulpal outcomes between stepwise and complete caries removal despite the marked difference in exposure rates. A strong limiting factor in the partial caries removal studies was the low quality of evidence regarding pulpal symptoms/health.
Preformed crowns were used in the no dentine removal primary teeth study (both occlusal and approximal lesions), and a bevelled intra-enamel occlusal resin restoration was used for the permanent teeth. The interventional techniques used to restore the teeth were very different, not allowing direct comparison of data. There were no differences in pulpal symptoms in the primary teeth study; the restorative outcomes for the intervention were much better than the complete caries removal. The permanent teeth study had no reports of pulpal symptoms, and there was no difference in the restorative outcome.

Most of the studies (apart from Innes) had shorter than desired follow-up periods, and this is a major shortcoming of the data set. What role the efficacy of restorative seal and initial pulpal health play in the long term is a question that needs to be answered.

This sound review highlights the lack of strong evidence for this important area of clinical practice. Possibly the use of practice-based research networks with well designed prospective trials could provide the data required to answer these questions which affect a clinician's practising methods every day, and possibly the outcomes for the patient.

David Manton

Department of Child Dental Health, Melbourne Dental School, The University of Melbourne, Melbourne, Australia.

1. Massler M. Pulpal reactions to dental caries. Int Dent / 1967; 17: 441-460.

2. Fusayama T. The process and results of revolution in dental caries treatment. Int Dent J 1997; 47: 157-166.

3. Alves LS, Fontanella V, Damo AC, Ferreira de Oliveira E, Maltz M. Qualitative and quantitative radiographic assessment of sealed carious dentin: a 10-year prospective study. Oral Surg Oral Med Oral Pathol Oral Radiol Endod 2010; 109: 135-141.

4. Peters MC, Bresciani E, Barata TJ, et al. In vivo dentin remineralization by calciumphosphate cement. J Dent Res 2010; 89: 286-291.

5. Thompson V, Craig RG, Curro FA, Green WS, Ship JA. Treatment of deep carious lesions by complete excavation or partial removal: a critical review. J Am Dent Assoc 2008; 139: 705-712.

6. Miyashita H, Worthington HV, Qualtrough A, Plasschaert A. Pulp management for caries in adults: maintaining pulp vitality. Cochrane Database Syst Rev 2007; 18: CD004484. DOI: 10.1002/14651858.CD004484

7. Aguilar $P$, Linsuwanont $P$. Vital pulp therapy in vital permanent teeth with cariously exposed pulp: a systematic review. / Endod 2011; 37: 581-587.

8. Ricketts D, Kidd E, Innes NPT, Clarkson JE. Complete or ultraconservative removal of decayed tissue in unfilled teeth. Cochrane Database Syst Rev 2006; 3: Art. No.: CD003808. DOI: 10.1002/14651858.CD003808.pub2.

Evidence-Based Dentistry (2013) 14, 74-75. doi:10.1038/sj.ebd.6400948 